\title{
Transport of hydrogen in metals with occupancy dependent trap energies
}

K. Schmid, ${ }^{1, a)}$ U. von Toussaint, ${ }^{1}$ and T. Schwarz-Selinger ${ }^{1}$

Max-Planck-Institut für Plasmaphysik, Boltzmannstraße 2, D-85748 Garching b. München Germany

Common diffusion trapping models for modeling hydrogen transport in metals are limited to traps with single de-trapping energies and a saturation occupancy of one. While they are successful in predicting typical mono isotopic ion implantation and thermal degassing experiments, they fail at describing recent experiments on isotope exchange at low temperatures. This paper presents a new modified diffusion trapping model with fill level dependent de-trapping energies that can also explain these new isotope exchange experiments. DFT calculations predict that even mono vacancies can store between 6 and $12 \mathrm{H}$ atoms with de-trapping energies that depend on the fill level of the mono vacancy. The new fill level dependent diffusion trapping model allows to test these DFT results by bridging the gap in length and time scale between DFT calculations and experiment.

a) Electronic mail: klaus.schmid@ipp.mpg.de 


\section{INTRODUCTION}

The commonly accepted picture of hydrogen $\mathrm{H}$ in metals like $\mathrm{Fe}^{7}$ or $\mathrm{W}^{11}$ (endothermal solution of $\mathrm{H}$ ) is that $\mathrm{H}$ is stored partially as solute in tetrahedral or octahedral sites and partially in defects. In the latter it is considered trapped and immobile until it de-traps into a solute site and continues to diffuse according to Fick's second law. The traps have fixed binding energies, different for each trap type and do not depend on the occupancy level of hydrogen in the trap ${ }^{7,8,11}$. The de-trapping step is thermally activated and is usually described by an Arrhenius type expression. This picture is very successful in describing experiments where hydrogen is loaded into the material by ion implantation ${ }^{9,11}$. In these experiments typically the sample is loaded at low temperature where little to no de-trapping occurs. Then the depth profile of the implanted $\mathrm{H}$, which decorates the trap sites, is measured and thus an estimate of the total trap site concentration in the material is obtained. Finally the sample is degassed with a linear heating ramp and the release rate of $\mathrm{H}$ is recorded, yielding a thermal desorption spectra (TDS). The TDS contains information on the different de-trapping activation energies and on the relative abundances of the different trap types. After modeling both the implantation and the TDS measurement with diffusion trapping codes one can infer a set of de-trapping energies and the trap site concentration depth profile. Using this information predictions can be made on how the material retains hydrogen species for instance as part of the fuel cycle in a magnetic confinement fusion experiment with metal walls ${ }^{9}$. From these experiments and modeling calculations it was concluded, that $\mathrm{H}$ is immobilized once trapped at a defect at low temperatures and can only be removed from the material by heating until significant de-trapping into the solute occurred. Net transport of $\mathrm{H}$ only occurs via diffusion when it is part of the solute population.

Recent experiments ${ }^{10}$ have shown that removal from trap sites is also possible at low temperatures where in the classic diffusion trapping picture no relevant de-trapping should occur at all. In these low temperature experiments W samples were initially loaded by ion implantation with Deuterium (D) and then subsequently implanted with H. In the classic diffusion trapping model all traps up to a certain diffusion limited depth are filled during the initial loading with D. Therefore the subsequently implanted H should just diffuse past these filled traps and be trapped beyond the initial depth profile deeper in the bulk. However the experiment showed that there was strong isotope exchange starting at the surface until 
way beyond the implantation range. This means that there is a process which allows newly implanted $\mathrm{H}$ to exchange with already trapped $\mathrm{D}$ which is not possible by Arrhenius type, thermally activated, de-trapping with fixed de-trapping energies as assumed in the classic diffusion trapping picture.

Here we propose a new diffusion trapping model based on fill level dependent de-trapping energies. The idea is that every trap site (e.g. mono vacancy) can store a certain number of hydrogen atoms and that the de-trapping energy de-crases with fill level. That means that above a certain fill level it is no longer energetically favorable to bind more hydrogen in that trap. The fill level dependence of the de-trapping energies allows to explain the low temperature isotope experiments as follows: During the D-loading phase all traps are filled to a maximum fill level in equilibrium with the solute $\mathrm{D}$. Once the $\mathrm{D}$ incident flux is switched off the highest fill levels are depopulated by de-trapping due to their low binding energies and the lack of solute D to repopulate them. This leaves the traps in the system filled with $\mathrm{D}$ to a level where the corresponding de-trapping energy is too high to de-trap at the current temperature. Once the $\mathrm{H}$ flux is turned on the traps are re-populated by the replenished solute population. This means that now the traps are filled with a mixture of $\mathrm{D}$ and $\mathrm{H}$ in equilibrium with the solute population. This equilibrium is a dynamic one: De-trapping from low binding energy high fill level states into the solute is compensated by trapping from the solute into the traps. Therefore there is an exchange between the mixture of D and $\mathrm{H}$ in the traps with the solute i.e. there is isotope exchange even at low temperature where the highest de-trapping energies (low fill levels) would normally not allow exchange with the solute.

This idea of fill level dependent de-trapping energies has been previously proposed by density function theory calculations ${ }^{4,6,12}$. They predict a fill level dependence of the de-trapping energy. However up to now there was no way to experimentally test their predictions since the experimental time and length scales are way beyond DFT capabilities. Therefore a diffusion trapping model has been developed with fill level dependent de-trapping energies that can directly use the DFT data. This allows to bridge the gap between DFT and experiment. This paper describes the newly developed fill level dependent diffusion trapping model in detail. Then test calculations are discussed that describe the implication of the fill level dependent de-trapping both on mono- and dual isotopic experiments. Finally the model is compared to real experimental data focussing on reproducing the experimental finding of 
isotope exchange at low temperatures.

\section{MODEL DESCRIPTION}

The idea is to describe the fill level dependent trapping using the same basic principles as in conventional diffusion trapping models ${ }^{7,11}$. Diffusion follows Fick's second law and trapping is controlled by Arrhenius type pre-factors. The main difference is how trapping and de-trapping into/from different fill levels is coupled. The coupling to the solute diffusion equations is essentially identical to that in current diffusion trapping models.

In contrast to current diffusion trapping models this model describes not the concentration of trapped $\mathrm{H}$-atoms in a given trap type, but the concentration of $\mathrm{H}$-atoms trapped in traps of a particular type and fill level.

The description of the model starts with expressions for the change in the number of particles before moving to concentrations.

The model is formulated for the general case of different trap types ti (e.g. Mono-vancies, dislocations etc.). However current calculations of the fill level dependence of the de-trapping energies are limited to simple Mono-vacancies. Therefore the example calculations are generally limited to a single ti $=1$. Still the ability of the model (or more accurately the code that implements it) to handle multiple trap types is demonstrated in an ad-hoc fit of experimental data in section IV.

\section{A. Model for mono-isotopic case}

The model describes the evolution of the number $\varepsilon_{k}^{t i}$ of H-atoms trapped in traps of type ti filled to level k. $\varepsilon_{k}^{t i}$ changes due to two basic processes:

- Trapping of a solute atom into a trap of type ti filled to level k-1 increases $\varepsilon_{k}^{t i}$ and correspondingly decreases $\varepsilon_{k-1}^{t i}$

- De-trapping from a trap of type ti filled to level $\mathrm{k}$ decreases $\varepsilon_{k}^{t i}$ and correspondingly increases $\varepsilon_{k-1}^{t i}$

These two processes result in a tight coupling between time evolution of adjacent trap fill levels. 
The trapping rate $\chi_{k}^{t i}(x, t)\left(s^{-1}\right)$ at a position $\mathrm{x}$ and time $\mathrm{t}$ of a solute H-atom into a trap of fill level $\mathrm{k}$ is proportional to:

- The number of solute atoms in lattice sites $n_{s}(x, t)$ a location $\mathrm{x}$ at time $\mathrm{t}$

- The probability $P_{k-1}^{t i}(x, t)$ that a neighboring site is a non saturated trap site with fill level k-1

- The success rate $\beta(T)\left(s^{-1}\right)$ of jumping one lattice distance $a_{0}(m)$ into the adjacent trap site

$P_{k-1}^{t i}(x, t)$ is determined by ratio of the number of traps of type ti that are filled to level $\mathrm{k}-1\left(\phi_{k-1}^{t i}(x, t)\right)$ to the total number of unoccupied sites (trap + solute $\left.=\Omega\right) \cdot \beta(T)$ is derived from the solute diffusion coefficient invoking "Einsteins-Relation". This results in eq. 1 for $\chi_{k}^{t i}(x, t)$.

$$
\begin{aligned}
\chi_{k}^{t i}(x, t) & =\beta(T) n_{s}(x, t) P_{k-1}^{t i}(x, t) \\
P_{k-1}^{t i}(x, t) & =\frac{\phi_{k-1}^{t i}(x, t)}{\Omega} \\
\beta(T) & =\frac{D^{\text {Solute }}(T)}{a_{0}^{2}} \\
D^{\text {Solute }}(T) & =\text { Solute diffusion coefficient }
\end{aligned}
$$

In an average over a large ensemble of atoms $\phi_{k-1}^{t i}(x, t)$ is simply given as the average of the trap occupancy $\varepsilon_{k-1}^{t i}$ as in eq. 2 .

$$
\phi_{k-1}^{t i}(x, t)=\frac{\varepsilon_{k-1}^{t i}(x, t)}{k-1}
$$

Eq. 2 is only valid for $k>1$ since the number of traps of type ti with fill level 0 (i.e. empty traps) has to be handled separately. It can not be derived from their average occupancy which is zero by definition. Their number is derived from the total number $N^{t i}$ of traps of type ti and from the values for $\phi_{k}^{t i}(x, t)$ with $k>0$ as in eq. 3 i.e. it simply equals the number of remaining unfilled traps. 


$$
\begin{aligned}
\phi_{0}^{t i}(x, t) & =N^{t i}(x, t)-\sum_{k=1}^{k_{\text {Max }}^{t i}} \phi_{k}^{t i}(x, t) \\
k_{\text {Max }}^{t i} & =\text { Maximum fill level of trap type ti }
\end{aligned}
$$

Basically eq. 3 describes the saturation of the system of traps with a certain maximum occupancy.

The de-trapping rate $\psi_{k}^{t i}(x, t)$ from traps of type ti filled to level $\mathrm{k}$ is given by a simple Arrhenius type equation as in eq. 4

$$
\begin{aligned}
\psi_{k}^{t i}(x, t) & =\alpha_{k}^{t i}(T) \varepsilon_{k}^{t i}(x, t) \\
\alpha_{k}^{t i}(T) & =\nu_{0} \operatorname{Exp}\left(\frac{-E_{k}^{t i}}{k_{B} T}\right) \\
E_{k}^{t i} & =\text { Activation energy to de-trap from trap type ti filled to level } \mathrm{k}
\end{aligned}
$$

To derive the equations for the time evolution of $\varepsilon_{k}^{t i}$ from the trapping (e.q. 1) and detrapping (eq. 4) rates the appropriate weight factors have to be derived. If a number of particles $\Delta \varepsilon_{k}^{t i}$ de-traps from level $\mathrm{k}, \varepsilon_{k-1}^{t i}$ is increased, on average, by $(k-1) \times \Delta \varepsilon_{k}^{t i}$ and $\varepsilon_{k}^{t i}$ is decreased on average, by $k \times \Delta \varepsilon_{k}^{t i}$. This can be seen by considering that each de-trapping step from level $\mathrm{k}$ produces a new trap site at level (k-1) filled with (k-1) atoms.

Similarly trapping $\Delta \varepsilon_{k}^{t i}$ atoms from solute into level $\mathrm{k}$, decreases level (k-1) by $(k-1) \times \Delta \varepsilon_{k}^{t i}$ and increases level $\mathrm{k}$ by $k \times \Delta \varepsilon_{k}^{t i}$. Based on this weighting considerations, the time evolution of $\varepsilon_{k}^{t i}$ can be written as in eq. 5 .

$$
\begin{aligned}
& \frac{\partial \varepsilon_{k}^{t i}}{\partial t}= k \times\left(\chi_{k}^{t i}(x, t)-\psi_{k}^{t i}(x, t)\right)-k \times\left(\chi_{k+1}^{t i}(x, t)-\psi_{k+1}^{t i}(x, t)\right) \\
& \text { for } 1<k<k_{M A X}^{t i} \\
& \frac{\partial \varepsilon_{k}^{t i}}{\partial t}=k \times\left(\chi_{k}^{t i}(x, t)-\psi_{k}^{t i}(x, t)\right) \text { for } k=k_{M A X}^{t i} \\
& \frac{\partial \varepsilon_{1}^{t i}}{\partial t}=\chi_{1}^{t i}(x, t)-\psi_{1}^{t i}(x, t)-1 \times\left(\chi_{2}^{t i}(x, t)-\psi_{2}^{t i}(x, t)\right) \text { for } k=1
\end{aligned}
$$


The total number of free sites $\Omega$ in eq. 1 can be written as in eq. 6 as the sum of free solute sites and free trap sites.

$$
\Omega=\left(N^{S}-n_{s}\right)+\sum_{t i=1}^{N^{T r a p s}}\left(N^{t i} k_{\text {Max }}^{t i}-\sum_{k=1}^{k_{M a x}^{t i}} \varepsilon_{k}^{t i}\right)
$$

With

$$
\begin{aligned}
N^{S} & =\text { Total number of solute sites } \\
n_{s} & =\text { Number of atoms on solute sites } \\
N^{\text {Traps }} & =\text { Total number of trap types ti }
\end{aligned}
$$

Assuming

$$
\begin{aligned}
& N^{S} \gg n_{s} \\
& N^{S} \gg N^{t i}
\end{aligned}
$$

it follows

$$
\Omega \approx N^{S}=\gamma N^{\text {Atoms }}
$$

With

$$
\begin{aligned}
N^{\text {Atoms }} & =\text { Number of host lattice atoms } \\
\gamma & =\text { Number of solute sites per host atom }
\end{aligned}
$$

Based on the approximate value for $\Omega$ in the limit of low site occupancy eq. 5 can be rewritten in terms of concentrations by dividing both sides of the equation by $N^{\text {Atoms }}$ as in eq. 7 


$$
\begin{aligned}
\frac{\partial C_{k}^{t i}}{\partial t}= & k\left(\frac{\beta(T)}{\gamma} C_{s} \frac{C_{k-1}^{t i}}{k-1}-\alpha_{k}^{t i}(T) C_{k}^{t i}\right)-k\left(\frac{\beta(T)}{\gamma} C_{s} \frac{C_{k}^{t i}}{k}-\alpha_{k+1}^{t i}(T) C_{k+1}^{t i}\right) \\
& \text { for } 1<k<k_{M a x}^{t i} \\
\frac{\partial C_{k}^{t i}}{\partial t}= & k \times\left(\frac{\beta(T)}{\gamma} C_{s} \frac{C_{k-1}^{t i}}{k-1}-\alpha_{k}^{t i}(T) C_{k}^{t i}\right) \text { for } k=k_{M a x}^{t i} \\
\frac{\partial C_{1}^{t i}}{\partial t}= & \frac{\beta(T)}{\gamma} C_{s}\left(\eta^{t i}-\sum_{k=1}^{k_{M a x}^{t i}} \frac{C_{k}^{t i}}{k}\right)-1 \times\left(\frac{\beta(T)}{\gamma} C_{s} \frac{C_{1}^{t i}}{1}-\alpha_{2}^{t i}(T) C_{2}^{t i}\right) \text { for } k=1
\end{aligned}
$$

With

$$
\begin{aligned}
C_{s} & =\frac{n_{s}}{N^{\text {Atoms }}} \text { Solute concentration } \\
C_{k}^{t i} & =\frac{\varepsilon_{k}^{t i}}{N^{\text {Atoms }}} \text { Concentration trapped in trap type ti to fill level k } \\
\eta^{t i} & =\frac{N^{t i}}{N^{\text {Atoms }}} \text { Concentration of type ti traps }
\end{aligned}
$$

The explicit dependencies on time $\mathrm{t}$ and location $\mathrm{x}$ have been omitted in eq. 7 for clarity. Eq. 7 can now be coupled to the solute diffusion transport equation (Fick's second law) as shown in eq. 8

$$
\begin{aligned}
\frac{\partial C_{s}(x, t)}{\partial t} & =D^{\text {Solute }}(T) \frac{\partial^{2} C_{s}(x, t)}{\partial^{2} x}+S(x, t)-\sum_{t i=1}^{N^{T r a p s}} \sum_{k=0}^{t_{M a x}^{t i}} \frac{\partial C_{k}^{t i}(x, t)}{\partial t} \\
S(x, t) & =\text { Source distribution }\left(s^{-1}\right)
\end{aligned}
$$

The only difference in eq. 8 to conventional diffusion trapping codes (see e.g. ${ }^{11}$ ) is the additional sum over the different fill levels.

Based on eq. 7 and eq. 8 the diffusion in a system with n-traps can be simulated by one partial differential equation (PDE) for the diffusive transport plus $\sum_{t i=1}^{N^{t r a p s}} k_{\text {Max }}^{t i}$ ordinary differential equations (ODE) for the trapping / de-trapping dynamics.

\section{B. Model for isotope exchange case}

The approach outlined in section II A for the mono-isotopic case can be extended to also include two isotopes which then allows to model isotope exchange experiments. In the 
following the concepts and equations are developed for isotope A without loss of generality. The corresponding equations for isotope B follow readily by exchanging A by B and vice versa. The quantity modeled in the isotope resolved case is $C_{m, k}^{t i}$ the concentration of an isotope of type $\mathrm{m}$ that is trapped in a trap of type ti with fill level $\mathrm{k}$. The fill level $\mathrm{k}$ now means that the sum of atoms of type A plus the sum of atoms of type B in the trap equals $\mathrm{k}$. In comparison to the model in section II A this distinction of two isotopes $(m \in\{A, B\})$ adds an additional coupling level: Changes in the amount of isotope $\mathrm{m}$ at level $\mathrm{k}$ affects the amount of both $\mathrm{A}$ and $\mathrm{B}$ at level $\mathrm{k}, \mathrm{k}-1$ and $\mathrm{k}+1$. Therefore $C_{A, k}^{t i}$ is now modified by the following processes:

- Trapping of a solute of type A or B into a trap of type ti filled to level k-1 increases $C_{A, k}^{t i}$ according to the fractional occupancy of level (k-1) with type A atoms $\left(\Lambda_{A, k-1}^{t i}\right)$ and accordingly decreases $C_{A, k-1}^{t i}$.

- De-trapping of an atom of type A or B from a trap of type ti filled to level k decreases $C_{A, k}^{t i}$ according to the fractional occupancy of level k with type $\mathrm{A}$ atoms $\left(\Lambda_{A, k}^{t i}\right)$ and accordingly increases $C_{A, k-1}^{t i}$.

The reason for introducing the fractional occupancy $\Lambda_{m, k}^{t i}$ is that since $\mathrm{k}$ is the occupancy of $\mathrm{A}+\mathrm{B}$ atoms, the actual occupancy of isotope A or B trapped at level $\mathrm{k}$ is not directly accessible. The fractional occupancy $\Lambda_{m, k}^{t i}$ can be derived as the ratio of the number $\varepsilon_{m, k}^{t i}$ of isotopes of type $\mathrm{m}$ trapped in trap type ti at fill level $\mathrm{k}$ to the number $\phi_{k}^{t i}$ of traps of type ti at fill level $\mathrm{k}$. Using a definition for $\phi_{k}^{t i}$ analogous to that in eq. 2 one obtains eq. 9.

$$
\begin{aligned}
\Lambda_{m, k}^{t i} & =\frac{\varepsilon_{m, k}^{t i}}{\phi_{k}^{t i}} \\
& =k \frac{\varepsilon_{m, k}^{t i}}{\varepsilon_{A, k}^{t i}+\varepsilon_{B, k}^{t i}} \\
\mathrm{Using} & \quad \varepsilon_{m, k}^{t i}=N^{A t o m s} C_{m, k}^{t i} \\
\Lambda_{m, k}^{t i} & =k \frac{C_{m, k}^{t i}}{C_{A, k}^{t i}+C_{B, k}^{t i}}
\end{aligned}
$$

Analogous to the mono-isotopic case the trapping $\chi_{m, k}^{t i}(x, t)$ and de-trapping $\psi_{m, k}^{t i}(x, t)$ rates for isotope $\mathrm{m}$ in trap type ti at fill level $\mathrm{k}$ can be written as in eq. 10 . 


$$
\begin{aligned}
& \chi_{m, k}^{t i}(x, t)=\frac{\beta(T)}{\gamma} C_{S}^{m}(x, t) \frac{C_{A, k-1}^{t i}(x, t)+C_{B, k-1}^{t i}(x, t)}{k-1}\left(s^{-1}\right) \text { for } k>1 \\
& \chi_{m, 1}^{t i}(x, t)=\frac{\beta(T)}{\gamma} C_{S}^{m}(x, t)\left(\eta^{t i}-\sum_{\tilde{k}=1}^{k_{M a x}^{t i}} \frac{C_{A, \tilde{k}}^{t i}+C_{B, \tilde{k}}^{t i}}{\tilde{k}}\right)\left(s^{-1}\right) \text { for } k=1 \\
& \psi_{m, k}^{t i}(x, t)=\alpha_{k}^{t i}(T) C_{A, k}^{t i}(x, t)\left(s^{-1}\right)
\end{aligned}
$$

The weighing factors that describe how $\chi_{m, k}^{t i}$ and $\psi_{m, k}^{t i}$ contribute to the time evolution of $C_{A, k}^{t i}$ are slightly more complex than for the mono isotopic case:

Trapping of $\Delta A \equiv \chi_{A, k}^{t i}$ atoms of type $\mathrm{A}$ and $\Delta B \equiv \chi_{B, k}^{t i}$ atoms of type $\mathrm{B}$ into traps of type ti at level (k-1) increases the concentration of atoms of type $\mathrm{A}$ at level $\mathrm{k}$ by:

$$
\Delta C_{A, k}^{t i}=(\Delta A+\Delta B) \times \Lambda_{A, k-1}^{t i}+\Delta A
$$

Similarly it decreases concentration of atoms of type A at level k-1 by:

$$
\left.\Delta C_{A, k-1}^{t i}=(\Delta A+\Delta B)\right) \times \Lambda_{A, k-1}^{t i}
$$

This means that the trapping of solute A and B converts a fraction of the traps with fill level(k-1), moving the atoms already stored there (according to their fractional occupancy) plus the newly trapped atoms to level $\mathrm{k}$.

In contrast detrapping $\Delta A \equiv \psi_{A, k}^{t i}$ atoms of type $\mathrm{A}$ and $\Delta B \equiv \psi_{B, k}^{t i}$ atoms of type $\mathrm{B}$ from traps of type ti at level $\mathrm{k}$ decreases the concentration of atoms of type $\mathrm{A}$ at level $\mathrm{k}$ by:

$$
\Delta C_{A, k}^{t i}=(\Delta A+\Delta B) \times \Lambda_{A, k}^{t i}
$$

Similarly it increases the concentration of atoms of type A at level k-1 by:

$$
\Delta C_{A, k-1}^{t i}=(\Delta A+\Delta B) \times \Lambda_{A, k}^{t i}-\Delta A
$$

This means that the de-trapping of $\mathrm{A}$ and $\mathrm{B}$ converts a fraction of the traps with fill level $\mathrm{k}$, moving the atoms already stored there (according to their fractional occupancy) minus the newly de-trapped atoms (lost to the solute) to level k-1. 
Based on eq. 11 to 14 the time evolution of the trapped concentration $C_{m, k}^{t i}$ can now be written similarly to eq. 7 but including the different weighing factors.

$$
\begin{aligned}
\frac{\partial C_{A, k}^{t i}}{\partial t}= & \left(\chi_{A, k}^{t i}+\chi_{B, k}^{t i}\right) \times \Lambda_{A, k-1}^{t i}+\chi_{A, k}^{t i}-\left(\chi_{A, k+1}^{t i}+\chi_{B, k+1}^{t i}\right) \times \Lambda_{A, k}^{t i}- \\
& \left(\psi_{A, k}^{t i}+\psi_{B, k}^{t i}\right) \times \Lambda_{A, k}^{t i}+\left(\psi_{A, k+1}^{t i}+\psi_{B, k+1}^{t i}\right) \times \Lambda_{A, k+1}^{t i}-\psi_{A, k+1}^{t i} \\
& \text { for } 1<k<k_{M a x}^{t i} \\
\frac{\partial C_{A, k}^{t i}}{\partial t}= & \left(\chi_{A, k}^{t i}+\chi_{B, k}^{t i}\right) \times \Lambda_{A, k-1}^{t i}+\chi_{A, k}^{t i}-\left(\psi_{A, k}^{t i}+\psi_{B, k}^{t i}\right) \times \Lambda_{A, k}^{t i} \text { for } k \equiv k_{M a x}^{t i} \\
\frac{\partial C_{A, 1}^{t i}}{\partial t}= & \chi_{A, k}^{t i}-\left(\chi_{A, 2}^{t i}+\chi_{B, 2}^{t i}\right) \times \Lambda_{A, k}^{t i}-\left(\psi_{A, 1}^{t i}+\psi_{B, 1}^{t i}\right) \times \Lambda_{A, 1}^{t i}+\left(\psi_{A, 2}^{t i}+\psi_{B, 2}^{t i}\right) \times \Lambda_{A, 2}^{t i}-\psi_{A, 2}^{t i} \\
& \text { for } K \equiv 1
\end{aligned}
$$

When inserting eq. 10 into 15 the sum $C_{A, k}^{t i}+C_{B, k}^{t i}$ appears both in the weighing factor $\Lambda_{m, k}^{t i}$ and in $\chi_{m, k}^{t i}$ and $\psi_{m, k}^{t i}$ and thus several factors cancel out, resulting in quite simple ODE's. Again the explicit dependencies of $C_{m, k}^{t i}$ and $\eta^{t i}$ on time t and location x have been omitted in eq. 15 for clarity. The coupling term to the solute transport is identical to the mono isotopic case except that there are now two solute transport equations, one for each isotope. Based on eq. 8 and eq. 15 the diffusion of two isotopes in a system with ntraps can be simulated by two partial differential equation (PDE) for the diffusive transport plus $2 \times \sum_{t i=1}^{N_{\text {traps }}} k_{\text {Max }}^{t i}$ ordinary differential equations (ODE) for the trapping / de-trapping dynamics.

\section{TEST CALCULATIONS WITHOUT DIFFUSION}

To better understand the difference between the classic diffusion trapping model and the here presented fill level dependent trapping it is instructive to compare the two approaches in $0 \mathrm{D}$ without solute diffusive transport. This can be readily achieved by removing the spacial dependence in eq. 7 and 15 and by imposing a certain time evolution of the solute concentration, thus mimicking the uptake and out-diffusion of solute from a surface.

To compare the result of the classic diffusion trapping model, with the results from the fill level dependent model, an equivalent set of classic trapping equations has to be setup. For a fill level dependent case with fill level dependent de-trapping energies $E_{k}^{t i}$ one classic 
trapping equation (see eq. 16 and 17) is setup with its fixed de-trapping energy equal to $E_{k}^{t i}$. This will yield the same amount of equations and concentrations in the result but without the inter-fill-level coupling terms. Also both models can store the same total amount of trapped atoms: In the classic case $\mathrm{k}$ trap types each with trap site concentration $\eta_{t i}$ and in the fill level dependent case one trap type at concentration $\eta_{t i}$ that can contain up to $k_{M A X^{-a t o m s . ~}}^{t i}$

For this model comparison a certain dependence of the de-trapping energy $E_{k}^{t i}$ (see eq. 4) on the fill level $\mathrm{k}$ is required. To simplify the results of the test calculations only three energies are used and only one trap type is assumed in the fill level dependent trapping equations: $E_{1}^{1}=1.41, E_{2}^{1}=1.14$ and $E_{3}^{1}=0.91 \mathrm{eV}$. The exponential pre-factor in the Arrhenius terms was set to $\nu_{0}=10^{13} \mathrm{~s}^{-1}$. The rational behind the choice of these energies is as follows. For a full model test there need to be at least three levels since $k \equiv 1$ and $k \equiv k_{\text {Max }}^{t i}$ require special treatment (see eq. 7. Also at least one level should show significant de-trapping at the calculation temperatures in order to allow isotope exchange at low temperatures.

\section{A. Mono-isotopic case}

For the mono isotopic case the corresponding 0D trapping equations to solve for the classic diffusion trapping model (see e.g. ${ }^{11}$ ) are summarized in eq. 16.

$$
\begin{aligned}
\frac{\partial C_{t i, k}^{T}(t)}{\partial t}= & \frac{\beta(T)}{\gamma} C_{S}(t)\left(\eta_{t i}-C_{t i, k}^{T}(t)\right)-\alpha_{t i}(T) C_{t i, k}^{T}(t) \\
C_{t i, k}^{T}(t)= & \text { Concentration of atoms trapped in trap type ti } \\
& \text { with de-trapping energy } E_{k}^{t i} \\
\eta_{t i}= & \text { Concentration of trap sites of type ti } \\
\alpha_{t i, k}(T)= & \text { Arrhenius de-trapping term with fixed activation energy } E_{k}^{t i} \\
C_{S}= & \text { Imposed solute concentration }
\end{aligned}
$$

For the model comparison eq. 16 and 7 were solved at a temperature of $500 \mathrm{~K}$ where two deepest chosen traps $\left(E_{1}^{1}\right.$ and $\left.E_{2}^{1}\right)$ exhibit little de-trapping whereas the shallowest trap $E_{3}^{1}$ shows significant de-trapping. In Fig. 1 the imposed solute concentration evolution as 
function of time is plotted: It steeply increases at around $1000 \mathrm{~s}$ and decreases to 0 at around $10^{5} \mathrm{~s}$ thus mimicking an implantation experiment where the solute rises quickly due to beam on and then decays slowly by out-diffusion after beam off. The trap concentration $\eta_{1}$ was assumed to be $10^{-4}$, a typical value found in implantation experiments ${ }^{11}$.

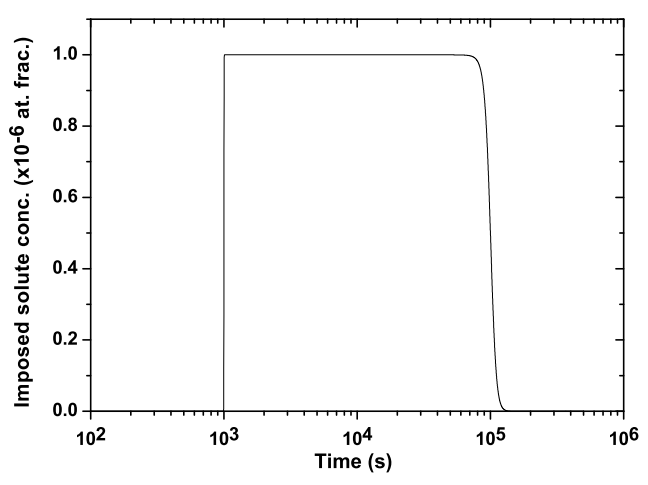

FIG. 1. The imposed solute concentration for the mono-isotopic test case calculations, mimicking the solute evolution during an ion beam implantation experiment

The resulting evolution of the trapped concentration for the classic model based on eq. 16 is shown in Fig. 2. As expected, as the solute is increased (left part of Fig. 2) the three traps are all filled to a level determined by the ratio of trapping to de-trapping. Therefore the shallowest trap $E_{3}^{1}$ is not entirely filled i.e. the trapped concentration is significantly less than $\eta_{1}$. As the solute source is decreasing (right part of Fig. 2), the de-trapping from the trap sites is no longer compensated by trapping from the solute and net loss of atoms occurs, the faster the lower their trapping energy.

It should be noted that at low enough temperatures no de-trapping would occur at all i.e. all the trapped concentration would stay constant even after the solute level is decreased to 0 .

For the fill level dependent trapping model based on eq. 7 the result of the test calculation is shown in Fig. 3.

In contrast to the classic model the different fill levels (corresponding to single traps in the classic case) are not all fully populated but are filled in stages: First the $\mathrm{k}=1$ level is filled as long as the solute concentration is low then as more solute is available and becomes trapped the $\mathrm{k}=1$ level is destroyed and converted into level $\mathrm{k}=2$ which finally as the traps saturate is converted to level $\mathrm{k}=3$. This conversion from $\mathrm{k}=2$ to $\mathrm{k}=3$ is only partial 


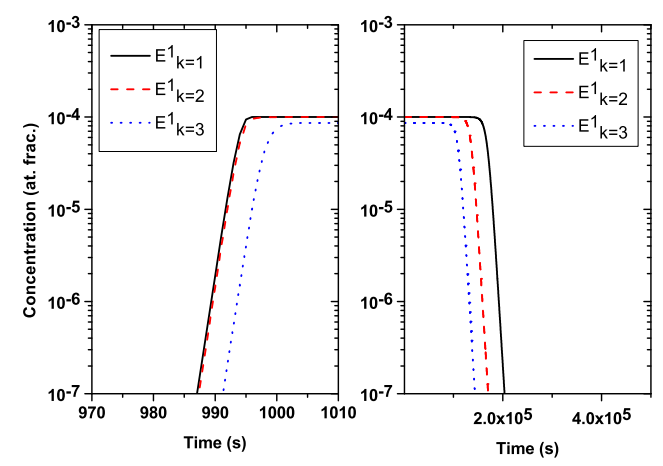

FIG. 2. The time evolution of the trapped concentrations for the classic model test calculation at $500 \mathrm{~K}$. The left figure shows the time around the onset of the increase in solute concentration. The right figure shows the time where the solute concentration decreases. (see also Fig. 1)

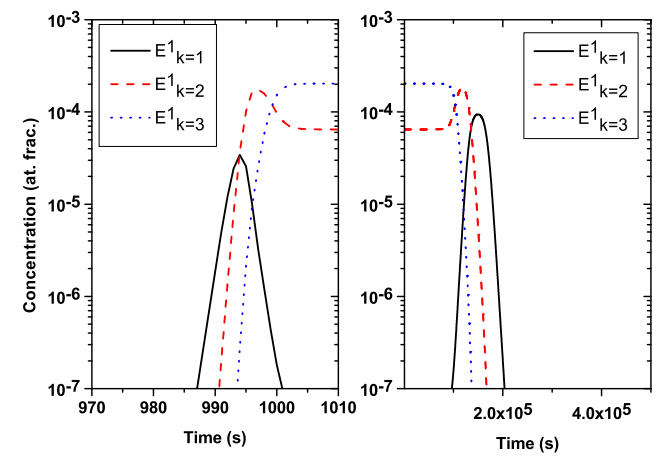

FIG. 3. The time evolution of the trapped concentrations for the fill level dependent trapping model test calculation $500 \mathrm{~K}$. The left figure shows the time around the onset of the increase in solute concentration. The right figure shows the time where the solute concentration decreases. (see also Fig. 1)

since due to the low de-trapping energy $E_{3}^{1}$ there is steady loss from $\mathrm{k}=3$ which in turn populates level $\mathrm{k}=2$. When the solute is decreased this afore described sequence is reversed and the level depopulate starting from $\mathrm{k}=3$ ending with depopulation of level $\mathrm{k}=1$.

While this stepwise populating and depopulating of levels in the fill level dependent trapping model at first looks very different from the classic picture, looking at the total amount stored in the traps in Fig. 4 shows that the over all difference is small. The principal shape of the two curves and thus the effective time scales are identical. The only difference is a slightly lower total amount in the fill level dependent case. This is due to the fact that most atoms are stored at the highest fill level (see also Fig. 3) which has the highest de-trapping 
rate and thus an equilibrium concentration $C_{1,3}^{T}$ which is lower than the maximum value of $k_{\text {Max }}^{1}(\equiv 3) \times \eta_{1}$. This is in contrast to the equivalent classic model which contains most of its atoms in traps at $E_{1}^{1}$ and $E_{2}^{1}$ which are both fully filled to their maximum concentration $\eta_{1}$. Thus this small difference is only present at elevated temperatures where significant de-trapping can occur from some $E_{k}^{t i}$. At low enough temperatures the models are identical.

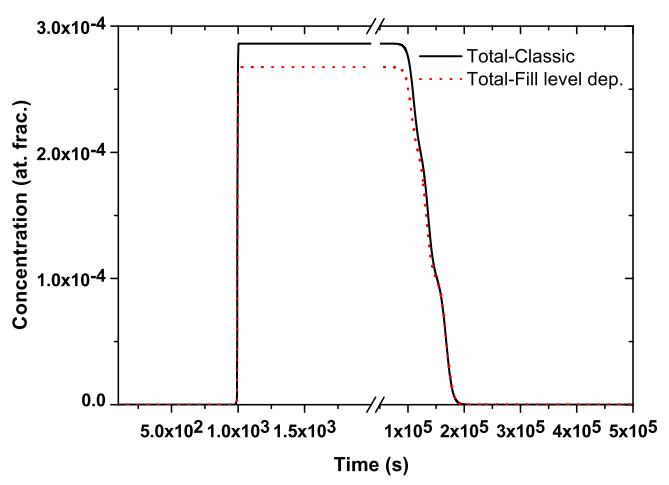

FIG. 4. Time evolution of the total trapped concentrations in all levels or individual traps with a linear concentration scale at 500K.

The fact that for the same binding energies the new fill level dependent model reproduces the classic model in the mono isotopic case is important, since the classic diffusion trapping models match a wide range of existing experimental data. The main reason for introducing the fill level dependence is the isotope exchange case at low temperatures for which the test calculations are shown in the next section.

\section{B. Isotope exchange case}

For the isotope exchange case the corresponding classic diffusion trapping model is summarized in eq. 17. Again only the equations for isotope A are shown the corresponding equations for isotope B follow readily as before. 


$$
\begin{aligned}
\frac{\partial C_{A, k}^{T, t i}(t)}{\partial t}= & \left.\frac{\beta(T)}{\gamma} C_{S}^{A}(t)\left(\eta_{t i}-C_{A, k}^{T, t i}(t)-C_{B, k}^{T, t i}(t)\right)-\alpha_{t i, k}(T) C_{A, k}^{T, t i}(t)\right) \\
C_{A, k}^{T, t i}(t)= & \text { Concentration of isotope A trapped in trap type ti } \\
& \text { with de-trapping energy } E_{k}^{t i} \\
\eta_{t i}= & \text { Concentration of trap sites of type ti } \\
\alpha_{t i, k}(T)= & \text { Arrhenius de-trapping term with fixed activation energy } E_{k}^{t i} \\
C_{S}^{A}(t)= & \text { Imposed solute concentration of isotope A }
\end{aligned}
$$

For the model comparison eq. 17 and 15 were solved at a temperature of $300 \mathrm{~K}$ where two deepest chosen traps $\left(E_{1}^{1}\right.$ and $\left.E_{2}^{1}\right)$ show no de-trapping whereas the shallowest trap $E_{3}^{1}$ still shows some de-trapping. In Fig. 5 the imposed solute concentration evolution of isotopes $\mathrm{A}$ and $\mathrm{B}$ as function of time is plotted: The solute concentration of isotope $\mathrm{A} C_{S}^{A}(t)$ steeply increases at around $1000 \mathrm{~s}$ and decreases to 0 at around $3 \times 10^{4} \mathrm{~s}$. This followed by an increase in $C_{S}^{B}(t)$ at $150 \times 10^{4} \mathrm{~s}$ which then subsequently decreases to 0 at $300 \times 10^{4} \mathrm{~s}$. This variation in $C_{S}^{A}(t)$ and $C_{S}^{B}(t)$ mimics an isotope exchange experiment where first the initially empty sample is loaded by isotope A and then, after a lag time of several hours is loaded with isotope B. The trap concentration $\eta_{1}$ was again assumed to be $10^{-4}$ and constant with depth. The resulting evolution of trapped concentrations of isotope A and B

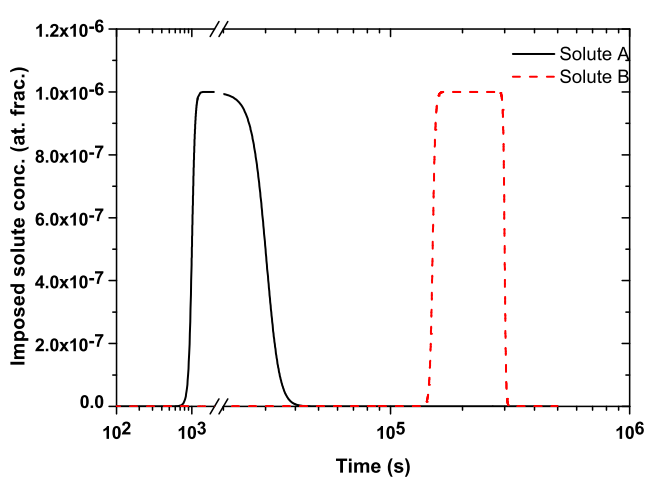

FIG. 5. The imposed solute concentration for the mono-isotopic test case calculations, mimicking the solute evolution during an ion beam implantation experiment at $300 \mathrm{~K}$

for the classic model based on eq. 17 is shown in Fig. 6. As $C_{S}^{A}(t)$ is increased all traps 
are filled and once $C_{S}^{A}(t)$ is reduced to 0 the atoms retained at $E_{1}^{1}$ and $E_{2}^{1}$ stay frozen in the traps whereas the atoms trapped at $E_{3}^{1}$ de-trap leaving empty traps behind. As $C_{S}^{B}(t)$ is now subsequently increased only traps at $E_{3}^{1}$ are available, so isotope $B$ only occupies the shallow traps and is thus lost when $C_{S}^{B}(t)$ is reduced back to 0 . This shows that in the

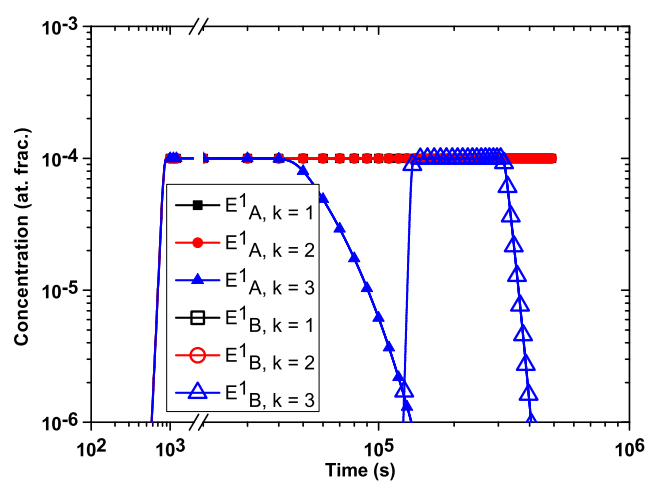

FIG. 6. Time evolution of the trapped concentrations of isotope A and B based on the classic model at $300 \mathrm{~K}$

classic picture isotope exchange is only possible at temperature where traps present in the sample de-trap the retained atoms, which is in contrast to recent experimental data.

For the fill level dependent trapping model based on eq. 15 the result of the test calculation is shown in Fig. 7. Similar to the mono isotopic case the different levels in the fill level

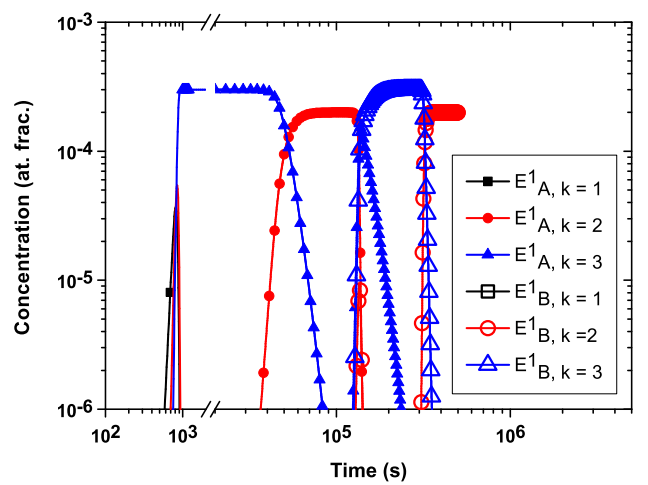

FIG. 7. The time evolution of the trapped concentrations for the fill level dependent trapping model test calculation at $300 \mathrm{~K}$.

dependent trapping model are filled in stages as the $C_{S}^{A}(t)$ is increased again ending up in an allmost fully populated level $\mathrm{k}=3$. Once $C_{S}^{A}(t)$ in decreased the shallow $E_{3}^{1}$ results 
in de-trapping from $\mathrm{k}=3$ resulting in a transfer into level $\mathrm{k}=2$ which stays frozen even after $C_{S}^{A}(t)$ has been reduced to 0 . As $C_{S}^{B}(t)$ is increased the levels are again filled in stages resulting in transfer into level $\mathrm{k}=3$ which now contains a mixture of isotope A and B. Since there is continuous de-trapping from level $\mathrm{k}=3$ and there is no remaining solute source of isotope $\mathrm{A}$, this results in a continuous loss of isotope $\mathrm{A}$ from the sample i.e. Isotope $\mathrm{A}$ is exchanged by isotope $\mathrm{B}$ at low temperatures where the classical picture does not predict significant exchange. After $C_{S}^{B}(t)$ is reduced to 0 again there is a transfer from $\mathrm{k}=3$ to $\mathrm{k}=$ 2 which is now only populated by isotope $\mathrm{B}$ which at the low temperature is frozen in the level $\mathrm{k}=2$ traps.

Looking at the total amounts of isotope A and B as function of time in Fig 8 the fundamental differences between the two models become apparent: In the classic case the trapped atoms are frozen whereas in the fill level dependent case trapping additional solute atoms of another isotope changes the de-trapping energy for the already trapped atoms, thus allowing them to de-trap even at low temperatures. As long as only isotope $\mathrm{A}$ is present the two models are

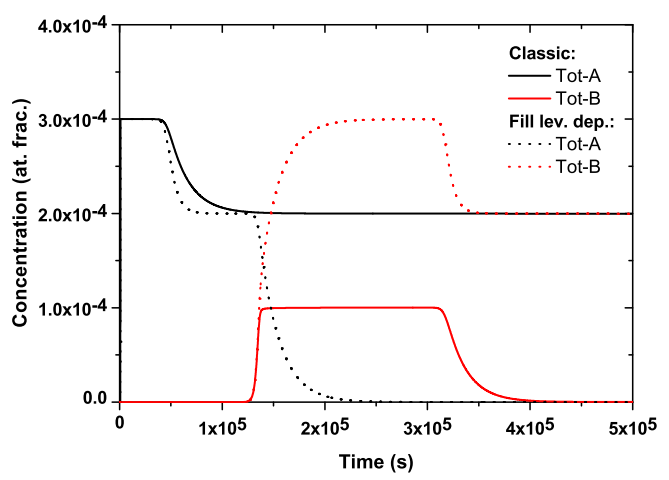

FIG. 8. The time evolution of the total trapped concentrations of isotope A and B in all levels or individual traps with a linear concentration scale.

essentially identical except for slightly different time scales: The fill level dependent model depletes slightly faster due to a higher de-trapping rate when all atoms are stored in the highest fill level which has the lowest de-trapping energy.

It should be noted that in the $0 \mathrm{D}$ treatment the exchanged isotope $\mathrm{A}$ is immediately lost from the sample whereas in reality it would add to $C_{S}^{A}(t)$ and be potentially re-trapped. The combined effect of trapping and diffusion will be treated in section IV. 


\section{COMPARISON TO EXPERIMENTAL DATA}

As explained in section III the main difference between the new fill level dependent model and the classic diffusion-trapping picture is expected in isotope exchange experiments. Therefore experimental data from a $\mathrm{D} / \mathrm{H}$ implantation experiment similar to ${ }^{10}$ was selected for comparison. In the experiment a recrystallized polycrystalline Tungsten (W) sample was bombarded with D at a flux of $10^{20} \mathrm{~m}^{-2} \mathrm{~s}^{-1}$ to a fluence of $10^{24} \mathrm{~m}^{-2}$ at $290 \mathrm{~K}$. After a waiting period of $\approx$ one day without implantation the sample was implanted with H-ions at a flux of $1.59 \times 10^{20} \mathrm{~m}^{-2} \mathrm{~s}^{-1}$ to a fluence of $0.75 \times 10^{24} \mathrm{~m}^{-2}$. The particle energy was $200 \mathrm{eV} /$ atom in both cases.

To model such an implantation experiment requires to include diffusion i.e. solve the full version of eq. 15 coupled to the solute transport via eq. 8. When diffusion is included, boundary conditions for the solute have to be chosen at the front and back surface of a sample with thickness $x_{\text {Max }}$. Currently diffusion limited boundary conditions are assumed which amounts to $C_{s}(0, t) \equiv C_{s}\left(x_{M a x}, t\right) \equiv 0$ (similarly also for the two isotope case). As additional input the solute diffusion coefficient is required. In this work the generally accepted values of Frauenfelder ${ }^{5}$ are used. The values in ${ }^{5}$ are for Hydrogen $(\mathrm{H})$. For isotope exchange models also a diffusion coefficient for Deuterium (D) is required. This value for D is derived from the value for $\mathrm{H}$ by scaling it with $\sqrt{\frac{m_{H}}{m_{D}}}=\frac{1}{\sqrt{2}}$.

Another critical input in the model are the de-trapping energies $E_{k}^{t i}$ (see eq. 4) as function of the fill level. Two sets of values $\left(\mathrm{Johnson}^{6}\right.$ and Ferro ${ }^{4}$ ) for de-trapping of $\mathrm{H}$ from a W mono vacancy are compared. Both are based on DFT calculations and are summarized in table I.

Both predict the same number of $6 \mathrm{H}$ atoms that can be stored in a $\mathrm{W}$ mono vacancy and

\begin{tabular}{|c|c|c|}
\hline $\mathrm{k}$ & Johnson $E_{k}^{t i}(\mathrm{eV})$ & Ferro $E_{k}^{t i}(\mathrm{eV})$ \\
\hline 1 & 1.41 & 1.43 \\
\hline 2 & 1.40 & 1.42 \\
\hline 3 & 1.14 & 1.25 \\
\hline 4 & 1.12 & 1.17 \\
\hline 5 & 0.91 & 1.11 \\
\hline 6 & 0.79 & 0.86 \\
\hline
\end{tabular}

TABLE I. Fill level dependence of the de-trapping energy in a $\mathrm{W}$ mono-vacancy according to Johnson and Carter ${ }^{6}$ and Ferro at $\mathrm{al}^{4}$ 
at first glance the predicted de-trapping energies are very similar. However the values by Johnson decrease quicker with fill level than those of Ferro. This results in more "active" trapped states at a given temperature. "Active" thereby refers to trapped states where there is significant net loss by de-trapping that is not compensated by trapping from the surrounding solute. This is in contrast to "static" trapped states from which, at a given temperature, there is no de-trapping. This can be made more quantitative based on the equilibrium solution of eq. 16 which yields the trapped concentration $C^{T, E q}$ in equilibrium with a given solute concentration $C_{S}$. As shown in eq. 18 the relative fill level (ratio of trapped concentration to trap site concentration) is determined by the relative magnitude of $\frac{\beta^{t i}}{\alpha_{k}^{t i}}$ and $\frac{1}{C_{S}}$ : If $\frac{\beta^{t i}}{\alpha_{k}^{t i}} \gg \frac{1}{C_{S}}$ all trap sites are filled independent on $C_{S}$ whereas if $\frac{\beta^{t i}}{\alpha_{k}^{t i}} \approx \frac{1}{C_{S}}$ only a fraction of sites are filled due to significant net loss from the traps by de-trapping.

$$
\frac{C_{t i, k}^{T, E q}}{\eta^{t i}}=\frac{\frac{\beta^{t i}}{\alpha_{k}^{t i}}}{\frac{1}{C_{S}}+\frac{\beta^{t i}}{\alpha_{k}^{t i}}}
$$

In Fig 9 the r.h.s of eq. 18 is plotted for a typical value of $C_{S}=10^{-7}$ and a temperature of $290 \mathrm{~K}$. For both the Johnson and Ferro data the equilibrium fill fraction of states $\mathrm{k}=1$ to 4 are equal to unity but states $\mathrm{k}=5$ and 6 are only partially filled. For the de-trapping energies from Johnson the equilibrium fill fraction of states $\mathrm{k}=5$ and 6 are significantly lower than for Ferro's energies resulting in more partially filled trap states in Johnson's case.

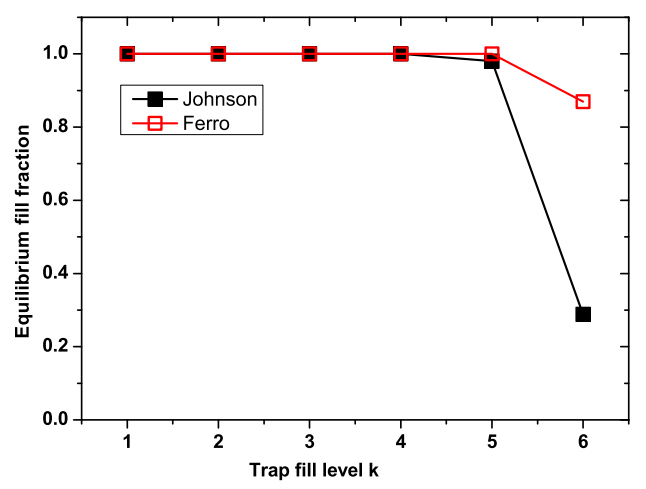

FIG. 9. Plot of the equilibrium fill level of a trap with de-trapping energies taken from the fill level $\mathrm{k}$ dependent de-trapping energies from Johnson and Ferro 
In the work by Ferro not just the de-trapping energies but also the Arrhenius frequency pre-factors are calculated as function of temperature. There is some strong variation at low temperature but for temperatures above $300 \mathrm{~K}$ the pre-factor is $\approx 10^{13} s^{-1}$. This value was also used for the calculations using Johnson's de-trapping energies.

Apart from the trap state (state $\equiv$ filled to level k) Arrhenius parameters also the depth distribution of trap sites $\eta^{t i}(x)$ is a key input parameter into the simulation. To estimate $\eta^{t i}(x)$ in a surface the near surface depth profiles of $\mathrm{D}$ or $\mathrm{H}$ implanted at low energies and temperature can be used. If the temperature is low enough such that no significant detrapping occurred, the D decorates all the trap sites and thus the depth profile corresponds to the trap site profile. Of course the so determined $\eta^{t i}(x)$ is the sum over all trap types ti. The relative abundance of each trap type can only be determined by modeling Thermal Desorption Spectra (TDS) see e.g. ${ }^{11}$. In the case of the fill level dependent model this trap site profile is only partially filled due to de-trapping from the high fill level states which are not occupied due to their low binding energies. Therefore the ratio of the depth profile to $\eta^{t i}(x)$ is a scaling factor $S_{\eta}$ which is $\leq k_{\text {Max }}^{t i}$, the maximum fill level. In the calculations here only one trap type is assumed with a fill level dependent de-trapping energy, so ti $=1$ and $S_{\eta}=k_{\text {Max }}^{t i}$ is assumed for simplicity. In Fig. 10 the chosen $\eta^{1}(x)$ is shown together with experimental D depth profile obtained after low temperature and energy implantation.

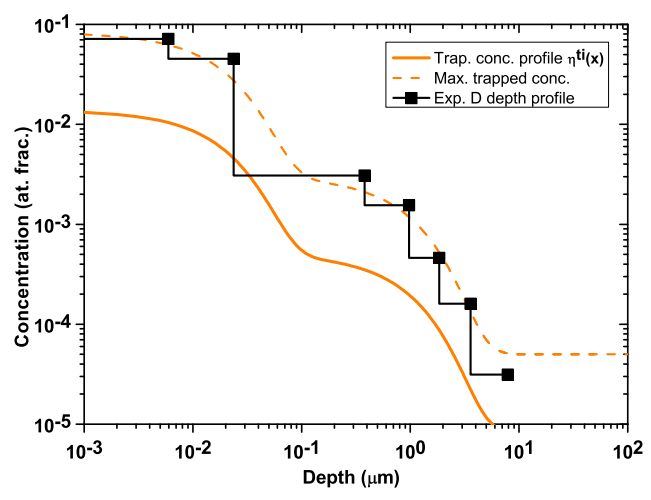

FIG. 10. Comparison of trap site concentration profile with D depth profile after low energy, low temperature implantation ( $\Leftrightarrow$ "Defect decoration by D")

In addition to the trap profile and trap energetics the source distribution $S^{M}(x)$ (see e.g. eq. 8) of isotope $\mathrm{M}$ has to be chosen according to the implantation conditions. $S^{M}(x)$ is well described by a Gauss shaped profile with its center at the mean projected range $R_{P}$ and a 
width based on the range straggling $\Delta R_{P}$. Using the Monte Carlo program SDTrim.SP ${ }^{3}$, which is a new version of TRIM.SP ${ }^{1,2} R_{P}$ and $\Delta R_{P}$ were calculated for $200 \mathrm{eV} \mathrm{D}$ and $\mathrm{H}$ ions. This calculation resulted in similar implantation range distributions for both $\mathrm{D}$ and $\mathrm{H}$ with mean projected range $R_{P}=6.2 \mathrm{~nm}$ and straggle of $\Delta R_{P}=3.4 \mathrm{~nm}$. The SDTrim.SP calculation also yielded a reflection yield of $R \approx 60 \%$. To account for reflection the incident ion flux was simply multiplied by R.

With the above choice of model input parameters (Trap energetics, Source distribution, Trap site depth profile) no free parameters are left.

To model the experimental sequence of D implantation, waiting period and $\mathrm{H}$ implantation the D, H flux time evolution depicted in Fig. 11 was used in the calculation. First the sample is implanted for $10^{4}$ sec. with $\mathrm{D}$ then the implantation flux is turned off and the sample is kept at constant conditions $(\mathrm{T}=290 \mathrm{~K})$ until at $80 \times 10^{4}$ sec. the $\mathrm{H}$ implantation flux is turned on.

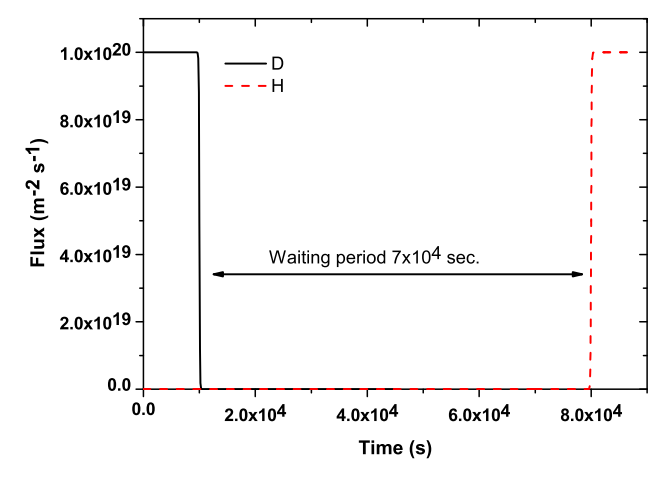

FIG. 11. Time evolution of D and $\mathrm{H}$ flux in the calculation

The implantation experiment can thus be divided into three phases:

- Loading: During the initial D implantation the traps in the sample are loaded with D.

- Degas: During the waiting period some net de-trapping from shallow trap states occurs and most of the solute is lost from the sample by outgassing.

- Isotope exchange: Finally the sample is implanted with $\mathrm{H}$ replenishing the fill level in the shallow trap states resulting in gradual isotope exchange of D by $\mathrm{H}$. 
The results of the full calculation (eq. 15 plus coupling to the solute transport via eq. 8) of all three phases is shown in Fig. 12 for the Ferro de-trapping energies and in Fig. 13 for the Johnson de-trapping energies. Both figures show the total D depth profile i.e. the sum over all trap fill levels states: $\sum_{k=1}^{k_{M a x}^{1}} C_{D, k}^{1}$, which is also what is experimentally available.

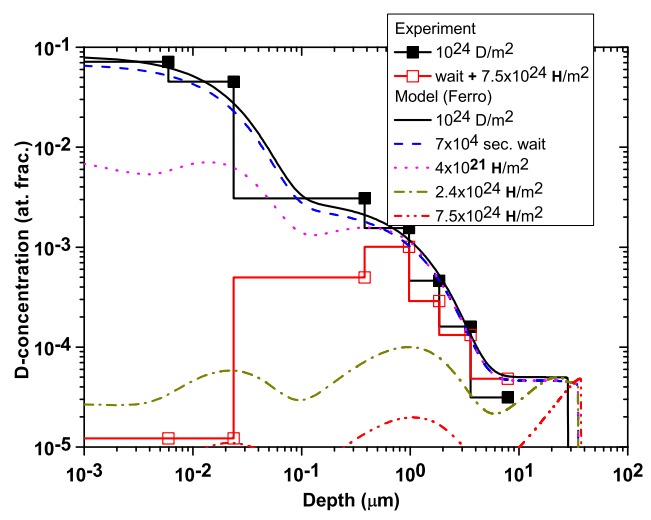

FIG. 12. Comparison of the calculated time evolution of the D depth profile with experimental data using de-trapping energies according to Ferro ${ }^{4}$.

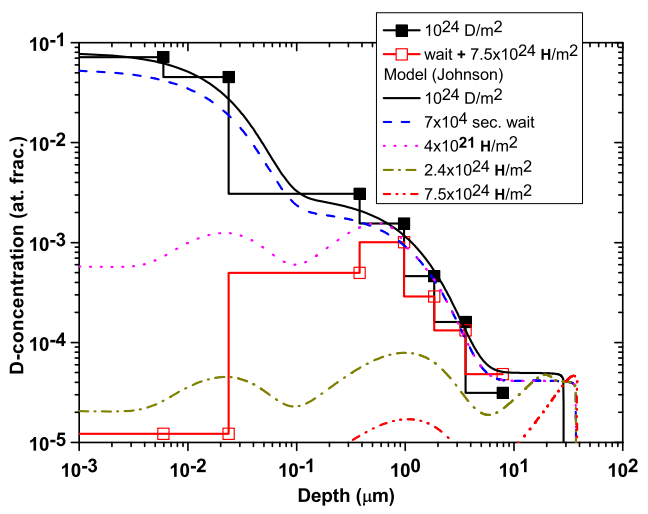

FIG. 13. Comparison of the calculated time evolution of the D depth profile with experimental data using de-trapping energies according to Johnson ${ }^{6}$

At first glance the calculation result based on the two de-trapping energies data sets look very similar. After the loading phase all trap fill levels are fully occupied thus matching the experimental depth profile. During the degas phase both show some loss from low binding energy fill levels, with the Johnson data set based calculation showing slightly more loss by de-trapping and outgassing as discussed above. During the isotope exchange phase strong 
exchange of D by $\mathrm{H}$ occurs in both cases. At low $\mathrm{H}$ fluences (graph at $4 \times 10^{21} \mathrm{H} / \mathrm{m}^{2}$ ) the calculation for the Johnson data set shows more isotope exchange that the Ferro data set based calculation. This can also be attributed to the lower de-trapping energies at high ( $\mathrm{k}$ $=5$ and 6) fill levels in Johnson's data set compared to Ferro's data.

These differences can be more clearly seen in Fig. 14 where the calculations based on the two de-trapping energy data sets are directly compared.

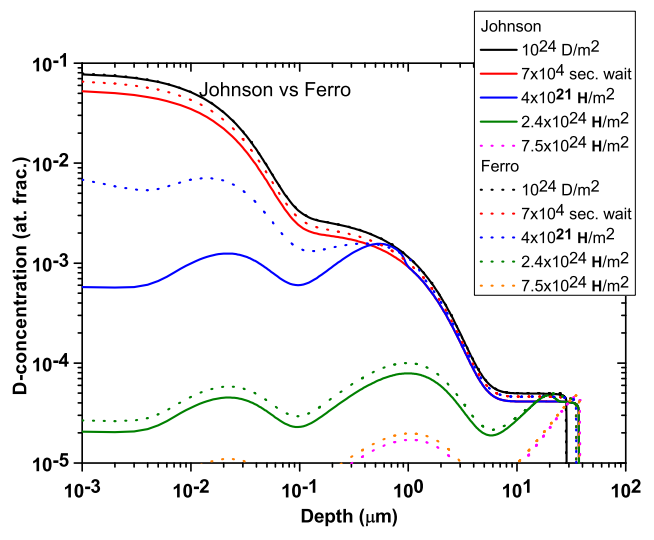

FIG. 14. Direct comparison of the D depth profile evolution calculated with the de-trapping energy data sets from Johnson and Ferro

Comparing either simulation to the experimental D depth profile obtained after the isotope exchange phase one can see that the model reproduces the general trends: Strong isotope exchange at low temperatures where classic diffusion trapping codes would predict essentially none at all. A closer look reveals that the isotope exchange in the model is significantly faster than in the experiment. The rate at which the isotope exchange takes place is determined by the speed at which the solute $\mathrm{H}$ diffusion front propagates into depth and the de-trapping rates from the shallow, high fill levels. At low temperatures and strong trapping the solute propagation is limited by the trap sites: Due to the high trapping rates $\beta$ the solute first has to saturate all trap states before it can progress further into depth. Thus the more empty trap states the slower the progress. During the isotope exchange phase most trap states are already filled by $\mathrm{D}$ thus the $\mathrm{H}$ solute diffusion front can propagate faster than the $\mathrm{D}$ solute diffusion front during the initial loading phase. In Fig 15 the position of the $\mathrm{H}$ solute diffusion front as function of time during the isotope exchange phase is shown. One can clearly see that based on Ferro's data the diffusion front moves faster than for Johnson's data. This is again due to the fact that for Johnson's data there is more de-trapping thus 
there are more empty trap states to fill thus slowing down solute propagation.

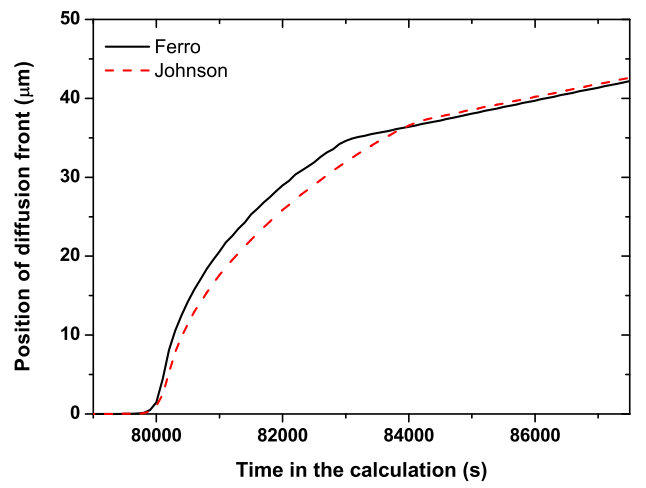

FIG. 15. Position of the $\mathrm{H}$ diffusion front during the isotope exchange phase for Ferro's and Johnson's de-trapping data sets

Since the de-trapping energy data sets from Johnson and Ferro are only valid for mono vacancies in $\mathrm{W}$ and since the polycrystalline material used in the experiment also contains other defects, mainly dislocations, choosing another set of de-trapping energies is justified. For instance one could assume different trap types, with different $E_{k}^{t i}$ and $k_{M a x}^{t i}$ values in the implantation zone and the bulk and thus different isotope exchange rates. To keep the number of free parameters as low as possible two traps $(t i \in\{1,2\})$ with two fill levels each $(k \in\{1,2\})$ are introduced with $\eta^{t i}(x)$ as in Fig. 16. $E_{k}^{t i}$ was chosen such that trap type 1 (only present in the implantation zone) would show fast isotope exchange whereas trap type 2 (only present beyond the implantation zone) would show less isotope exchange. The so chosen values are summarized in table II. The comparison of the so calculated total D depth profile to the experimental D depth profile is shown in Fig. 16. The model reproduces the experimental data well. Both the initial D loading phase and the isotope exchange phase are reproduced reasonably well.

This example of two trap types with adjusted $E_{k}^{t i}$ is of course a rather ad-hock approach, but

\begin{tabular}{|c|c|c|}
\hline $\mathrm{ti}$ & $\mathrm{k}$ & $E_{k}^{t i}(\mathrm{eV})$ \\
\hline 1 & 1 & 1.41 \\
\hline 1 & 2 & 0.79 \\
\hline 2 & 1 & 1.41 \\
\hline 2 & 2 & 0.95 \\
\hline
\end{tabular}

TABLE II. The fill level dependent trap energies used in the ad-hock fit to the experimental data 


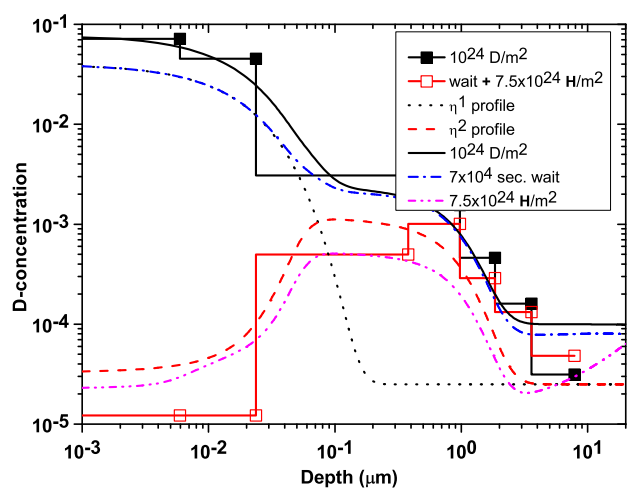

FIG. 16. Comparison of ad-hock fit of experimental data with two fill level dependent traps. Also shown are the assumed $\eta^{t i}$ profiles

shows that the model can reproduce the experimental data. Further progress in fundamental modeling is required to also supply $E_{k}^{t i}$ data sets for other types of defects. The new fill level dependent diffusion trapping model now allows to test these data sets by providing a means for comparison with real experimental data.

\section{SUMMARY}

The recent experimental finding of isotope exchange during ion implantation experiments at low temperatures in $\mathrm{W}$ can not be explained by classic diffusion trapping models since they predict hydrogen to be "frozen" in their trapped state at these temperatures. Following predictions from DFT, that trap sites in metals like W can contain multiple hydrogen atoms with fill level dependent de-trapping energies $E_{k}^{t i}$, a modified diffusion trapping model was developed that can describe fill level dependent trapping. This new model allows, for the first time, to test the DFT predictions on the fill level dependence of de-trapping energies against real experimental data.

First test calculations based on available DFT data for $E_{k}^{t i}$ have shown that while the model qualitatively reproduces the experiment, the DFT data for single vacancies is not yet sufficient to describe the $E_{k}^{t i}$ for all the trap types contributing in current experiments. A fit of an available experimental data set with an ad-hock choice of $E_{k}^{t i}$ revealed that the newly developed model can match the experimental data quite. More effort is need to yield sound for $E_{k}^{t i}$ not just for mono-vacancies but also for other defects (e.g. dislocations). This may 
however require to move from DFT to molecular dynamics methods due to the extended size of such defects.

\section{ACKNOWLEDGMENTS}

This work was supported by EURATOM and carried out within the framework of the European Fusion Development Agreement. The views and opinions expressed herein do not necessarily reflect those of the European Commission. 


\section{REFERENCES}

${ }^{1}$ Biersack, J. and Eckstein, W., Appl. Phys. A 34, 73 (1984).

${ }^{2}$ Eckstein, W., Computer Simulation of Ion-Solid Interactions (Springer Verlag, 1991).

${ }^{3}$ Eckstein, W., Dohmen, R., Mutzke, A., and Schneider, R., Report IPP, Garching 12/3 (2007).

${ }^{4}$ Fernandez, N., Ferro, Y., and Allouche, A., to be published (2014).

${ }^{5}$ Frauenfelder, R., J. Vac. Sci. Technol. 6(3), 388 (1969).

${ }^{6}$ Johnson, D. F. and Carter, E. A., J. Mater. Res. 25 No. 2, 315 (2010).

${ }^{7}$ Krom, A. H. M. and Bakker, A., METALLURGICAL AND MATERIALS TRANSACTIONS B 31B, 1475 (2000).

${ }^{8}$ Longhurst, G. R. and Ambrosek, J., Fusion Science and Technology 48, 468 (2005).

${ }^{9}$ Ogorodnikova, O. V., Roth, J., and Mayer, M., J. Appl. Phys 103, 34902 (2008).

${ }^{10}$ Roth, J., Schwarz-Selinger, T., Alimov, V., and Markina, E., J. Nucl. Mat. 432, 341 (2013).

${ }^{11}$ Schmid, K., Rieger, V., and Manhard, A., Journal of Nuclear Materials 426, 247 (2012).

${ }^{12}$ Sun, L., Jin, S., Li, X.-C., Zhang, Y., and Lu, G.-H., J. Nucl. Mat. 434, 395 (2013). 